\title{
Examen critique de la notion de fétiche à partir du cas evhé
}

A Critical Examination of the Notion of Fetish Using Ewe Data (Togo)

\section{Albert de Surgy}

\section{OpenEdition}

1 Journals

Édition électronique

URL : http://journals.openedition.org/span/1081

DOI : 10.4000/span. 1081

ISSN : 2268-1558

Éditeur

École pratique des hautes études. Sciences humaines

\section{Édition imprimée}

Date de publication : 1 septembre 1987

Pagination : 263-304

ISSN : 0294-7080

\section{Référence électronique}

Albert de Surgy, «Examen critique de la notion de fétiche à partir du cas evhé », Systèmes de pensée en Afrique noire [En ligne], 8 | 1987, mis en ligne le 07 octobre 2013, consulté le 01 mai 2019. URL : http:// journals.openedition.org/span/1081; DOI : 10.4000/span.1081 


\title{
EXAMEN CRITIQUE DE LA NOTION DE FETICHE
}

\section{A PARTIR DU CAS EVHE}

\author{
par
}

\section{Albert de Surgy}

Les Africains que nous connaissons ne se contentent pas d'adorer humblement des divinités, invitées à venir siéger sur des autels pour y écouter des prières et y recevoir des oblations. Ils se dépensent pour fabriquer puis entretenir des puissances leur permettant de dominer par euxmêmes le cours des événements comme de faire pression sur de nombreux agents immatériels extérieurs entrant en jeu dans les processus de leur détermination.

On ne saurait bien rendre compte de leurs religions en ignorant de telles pratiques ou en les marginalisant par rapport à de plus louables efforts d'union de l'âme à Dieu lui-même par le canal des êtres et des principes de l'univers qui lui sont intégralement subordonnés. Une manipulation quasi technique et intéressée des forces cachées de la nature fait en effet chez eux excellent ménage avec un authentique souci de sanctification de soi-même et du monde par participation enthousiaste à l'oeuvre du Créateur. La confrontation avec de telles forces caractérise une voie d'élévation spirituelle sans rupture de continuité, parvenant à mobiliser l'énergie humaine depuis les plus bas niveaux de stagnation de la conscience jusqu'aux plus hauts lieux de son raffinement.

Dans ces conditions, ne convient-il pas de réexaminer ce sur quoi l'appellation de fétichisme a fini par attirer le mépris, et 
de le remettre à sa juste place au sein d'ensembles cohérents de conduites symboliques d'autant plus dignes d'intérêt que nous y trouvons simultanément mis en évidence un Dieu unique trônant au sommet ou au coeur de l'univers, une pluralité de dieux, de divinités, d'ancêtres et d'esprits, ainsi qu'une grande variété d'objets chargés et nourris d'énergie subtile ? Que les prétendus stades fétichiste, animiste, polythéiste et monothéiste, au travers desquels les sociétés humaines se dégageraient d'une primitive obscurité intellectuelle, coexistent de la sorte, aussi vivaces les uns que les autres et se renforçant mutuellement, porte notamment condamnation des conceptions évolutionnistes ayant été propagées par Auguste Comte.

Mais ne vaudrait-il pas mieux, du même coup, se débarrasser du terme de fétiche ? Une connotation péjorative d'obscurantisme et de tromperie y reste en effet attachée et les multiples usages qui en ont été faits ne manquent pas de prêter à confusion. Employé à l'origine par les Portugais pour désigner les supports matériels (feitiços) d'activités magiques employés le long de l'ancienne Côte de Guinée pour tourner le sort à son avantage ou au désavantage de ses ennemis, il n'a que trop servi, depuis lors, au point que la plupart des ethnologues ont fini par l'exclure de leur vocabulaire, à nous masquer des réalités que 1 'on désirait plier à la justification d'idéologies, de caractère réductionniste ou impérialiste, éprouvant le besoin, pour se faire valoir, de dénoncer les erreurs, l'insanité ou le primitivisme d'autrui.

On peut supposer qu'après une exploration objective de l'ensemble du domaine auquel il est susceptible de conduire, des spécialistes de bonne foi parviendraient sans grande difficulté à un accord pour en formuler enfin une définition précise. Encore faudrait-il que cette définition ne lui fixe pas de limites trop arbitraires et s'avère utile à l'analyse des observations effectuées. On verra pourquoi je me permets d'en douter.

Tout en reconnaissant l'impossibilité de le rayer purement et simplement du vocabulaire, je me propose d'expliquer ci-dessous pourquoi il me parất impropre à l'inventaire et à l'interprétation des nombreux objets d'activité magico-religieuse que 1 'on est pourtant fortement tenté d'appeler ainsi chez les Evhé de la côte togolaise 
et ghanéenne. En compensation je suggérerai d'aborder l'étude de tels objets en ayant à l'esprit d'autres notions qui présentent l'avantage d'être transposables à d'autres civilisations.

\section{L'UNIVERS RELIGIEUX DES EVHE}

En tant qu'ethnologues, nous devons éviter de nous embourber dans une glose de notions ayant été élaborées, si rapidement qu'elles en ont paru objectives, dès les premiers contacts avec des peuples lointains. Nos efforts méritent plutôt d'être consacrés à une analyse des faits auxquels ces notions renvoient. Une observation judicieuse et soutenue de tels faits amène immanquablement à réviser les notions auxquelles nous les rattachions a priori ou à en adopter d'entièrement nouvelles. Je commencerai done par une présentation générale du système religieux des Evhé avant de me demander s'il accorde ou non droit de cité à des objets de vénération pouvant à bon droit être nommés f'étiches.

\subsection{Le monde de 1 'origine et la programmation naturelle des phénomènes qui $s^{\prime} y$ rattachent}

Selon les conceptions traditionnelles des Evhé, tout ce qui vient se manifester à la surface de la terre y est envoyé depuis un monde lointain de l'origine de toutes choses, situé sous nos pas dans un abîme radicalement invisible, débouchant, par delà la ligne d'horizon, du côté où le soleil se lève.

Il s'y trouve un magasin universel des possibilités ne contenant nullement, sous forme codée ou en image, tous les phénomènes susceptibles de se produire, mais seulement les éléments permettant à des esprits (luvho) engagés par Dieu (Mawu) comme tâcherons d'élaborer eux-mêmes, comme fruits d'une existence prénatale, les germes de ceux dont ils prendront ensuite à coeur d'assurer la réalisation.

On donne au lieu mythique de cette élaboration le nom d'amedzophe, "Iieu (phe) d'où provient (dzo) la personne humaine (ame)", ou de 
nolime, "chez les âmes non incarnées ou désincarnées", en tant qu'il est habité par les âmes humaines avant qu'elles ne prennent corps ou après la désagrégation de celui-ci, ou encore de bome, "dans le champ-clairière de culture", car I'homme y aurait ensemencé comme dans un champ lointain aménagé en pleine forêt les germes des phénomènes dont la responsabilité lui est attribuée et qui y prennent racine comme les plantes au sein d'une terre nourricière. On l'estime en outre placé sous le gouvernement bienveillant d'une génitrice universelle appelée Niolimeno ou Bomeno ("la mère de ronolime ou de bome").

De même que les productions végétales sont soumises aux cycles saisonniers, l'activation des germes des phénomènes est soumise à une loi naturelle fixée une fois pour toutes par Dieu, associée à la régularité du mouvement des corps célestes, que des entités divines subordonnées à lui, agissant en intendantes de son domaine, se trouvent chargées de faire respecter.

En tant que l'homme est surdéterminé par la culture à laquelle il appartient, l'activation des germes des phénomènes dont il est l'acteur dépend cependant aussi, dans une très large mesure, de I'héritage de ses ancêtres. Cet héritage lui parvient de trois manières : en ligne patrilinéaire pour ce qui concerne essentiellement le respect des coutumes et la puissance de rassemblement, d'unification et de commandement des hommes, en ligne matrilinéaire pour ce qui concerne essentiellement la force physique, le bien-être et le mode de sensibilité, enfin par une troisième voie indépendante de l'appartenance à une lignée précise, allant cette fois de mâ̂tre à disciple, pour ce qui concerne l'expression particulière de la vitalité et les aptitudes professionnelles.

Alors que les deux premières voies représentent surtout l'arbitraire de la tradition, en rapport toutefois avec un principe général d'harmonie et un principe de générativité comme de contamination, la troisième le rattache à des puissances universelles d'expression des capacités innées que sont les génies du destin (se) et les vodu, spécialisés dans la mise en valeur de certains types de "nature" (dzodzome), c'est-à-dire d'invisibles champs d'implantation de 1 'âme. 
La production des événements qui intéressent les hommes est donc non seulement placée sous le contrôle de gestionnaires divins de la création (Se et vodu), mais aussi sous celui d'ancêtres agissant dans de nombreux cas en relation directe avec eux.

\subsection{La possibilité pour 1 'homme de faire appel à la source de toute chose}

S'étant souverainement éloigné de son oeuvre, Dieu (Mawu) a accordé aux représentants divins de sa volonté mis en poste sur son domaine, mais aussi bien aux ancêtres admis auprès d'eux, une très large autonomie.

$\mathrm{Ce}$ sont des âmes d'ancêtres qui établissent une liaison entre le monde des hommes et le monde de l'origine. En effet toute âme humaine est constituée par l'appariement de deux sortes d'esprits (luvho) : un esprit vital (agbe luvho), ou ombre dense, chargé de maintenir le corps en vie, et un esprit imaginant, ou esprit de mort (ku luvho), ou ombre longue, chargé d'entretenir parallèlement un univers individuel de représentations mentales. Du vivant de l'inåividu ces deux esprits demeurent attelés côte à côte à la même tâche, mais ils ne sont plus astreints après la mort à une telle proximité. L'esprit imaginant demeure invisiblement sur terre aux côtés de ses descendants pour prendre connaissance de ce qui leur arrive, écouter leurs prières et recevoir leurs offrandes. Cependant l'esprit vital d'un "bon mort" regagne sa place au pays de l'origine auprès du principe vital (kla) ayant présidé à son envoi au monde. Il y rejoint l'esprit vital de l'ancêtre (amedzoto-) qui l'avait accompagné jusqu'au seuil du monde visible afin qu'il naisse au sein de tel lignage, du ventre d'une femme originaire de telle ou telle famille.

Pour se rendre utiles à leurs descendants, les ancêtres instituent au sein de leur propre société des groupes plus ou moins étroitement spécialisés dans la surveillance de l'amorçage de telle ou telle sorte d'événements, ainsi que dans des interventions auprès des représentants de Dieu pour obtenir l'envoi au monde de faveurs particulières. 
Ce sont ces groupes, pour beaucoup temporaires, qui sont identifiés à des divinités. L'homme a la possibilité de s'adresser à eux pour leur demander de modifier ou d'achever de déterminer en sa faveur la programmation naturelle des événements. Outre les offrandes au moyen desquelles il se les rend propices, il leur doit des sacrifices en échange des travaux, assimilables à des actes de "culture", qu'ils accomplissent pour luj à cet effet dans les lointains. S'il ne s'acquitte pas envers eux de ses dettes sacrificielles, ils n'hésitent pas à le rappeler à l'ordre en lui infligeant divers désagréments pouvant aller jusqu'à la mort. Cette habitude de retournement contre les bénéficiaires de leurs interventions leur vaut le titre général de tro (qui signifie changer sa route, tourner, revenir). Toutefois ceux d'entre eux dont les affiliés se recrutent en suivant la troisième voie de transmission mentionnée plus haut, et qui mettent les vivants en relation avec les principes vitaux, reçoivent plutôt désormais le nom (d'origine dahoméenne) de vodu.

Quelle qu'en soit la catégorie, il s'agit d'entités transcendantes que l'homme peut certes supplier, voire même menacer de ne plus prendre en considération si elles l'abandonnent à son sort, mais auxquelles il ne peut en aucun cas forcer la main. Il leur demeure irrévocablement assujetti et s'humilie devant elles en tremblant constamment d'espoir (en leurs faveurs) ou de crainte (d'être sanctionné pour ne point leur rendre leur dû).

\subsection{Le processus d'actualisation des événements ayant été amorcés}

Entre le moment où un événement est amorcé par activation de son germe et celui où il se concrétise s'écoule une durée comparée à celle qui sépare la conception d'un enfant dans le sein de sa mère du moment de son accouchement au grand jour. Entre le lieu depuis lequel il est émis et celui de son apparition sur terre il est imaginé suivre un trajet analogue à celui imposé aux fluides nourriciers par le cordon ombilical qui unit le placenta (symbole de la terre originelle) au foetus. 
Tout au long de ce trajet, c'est-à-dire aussi longtemps qu'un événement se prépare dans l'ombre, il n'est pas encore absolument déterminé. Il suffit qu'une influence impondérable le pousse à bifurquer à tel ou tel moment dans une direction plutôt qu'une autre pour entrainer d'importantes modifications de son aspect final, provoquer en quelque sorte son avortement ou le transformer en catastrophe. Or rien n'interdit de penser qu'une force ou une intelligence n'oriente pas invisiblement à ce moment précis ce qui nous paraît ensuite engendré par le hasard.

Pourvu que globalement sa probabilité n'en soit pas significativement modifiée, nul ne pourra jamais prouver en effet qu'un esprit est intervenu ou n'est pas intervenu dans le surgissement au moment opportun d'un micro-événement de nature à aiguiller l'avenir sur un chemin sans retour ou à provoquer à terme, par un enchaînement de conséquences, un bouleversement considérable. Il suffit que l'esprit en question ait eu la possibilité d'équilibrer son intervention décisive par une intervention peu conséquente en sens inverse pour que nul ne s'en aperçoive; en effet il aura seulement réarrangé alors à sa convenance une suite "naturelle" d'occurrences en une autre suite équiprobable, et par conséquent semblablement "naturelle".

C'est ainsi que les Evhé, sans offenser le moindrement la raison, imaginent, au sein de l'espace intermédiaire entre le monde de l'origine et le monde sensible objectivé, une multitude de causes agissantes complémentaires, à distinguer de tous les agents (ancêtres et divinités) qui contribuent supérieurement de plein droit à la programmation de l'activation des germes de phénomènes. Elles ne peuvent modifier cette fois que les conditions de gestation de ces phénomènes, mais sont en mesure de multiplier de la sorte les chances et les malchances qui affectent les individus.

Il est clair qu'entre une décision et I'exécution de cette décision s'intercale une période de rassemblement des moyens et de mobilisation des énergies. Un événement décidé est loin d'être encore réalisé. De ce point de vue, alors qu'ancêtres et divinités ont seuls le pouvoir d'intervenir au niveau où en est ordonnée la mise en route, d'autres "puissances" sur lesquelles cette fois les hommes ont prise, que ce soit par contrainte, par ruse ou par négociation, 
entrent en jeu au niveau de la concertation spirituelle nécessaire à son agencement particulier.

Alors que l'introduction naturelle d'un événement en position de départ ne consomme aucune parcelle de l'énergie nécessaire pour porter le monde à l'existence en l'engageant puis en continuant de le pousser sur le parcours menant du niveau de sa conception à celui de sa naissance (en d'autres termes de son état en puissance à ce qu'il nous apparaît en acte), et alors que toute modification de la programmation des départs des futurs événements, bien que devant être compensée par un sacrifice, n'en consomme pas davantage, la mise en route effective des événements et leur propulsion jusqu'à terme, comme toute modification de leur parcours, en réclame impérieusement.

Indépendamment des modifications, plus riches d'effets, des causes finales (conscientes ou inconscientes) de leurs activités, il n'est possible aux hommes d'intervenir au niveau intercalaire entre ce qui subsiste en puissance et leur expérience vécue qu'en épuisant les impulsions eréatrices attachées aux événements en gestation ou en déformant, à l'aide de facteurs d'incitation du même ordre, non intégrés à l'univers perceptible, les lignes de force qu'ils empruntent pour approcher de leur réalisation.

\subsection{L'intervention de divinités ou d'esprits indépendants au niveau d'un tel processus}

Pour être pleinement efficaces dans ces conditions, les divinités doivent se donner les moyens d'aider à l'acheminement jusqu'au monde, en écartant les incidents de parcours, des événements dont elles souhaitent ou ont parfois même amorcé dans le secret la réalisation.

A cet effet, dans la plupart des cas, elles accueillent à leur service des âmes humaines ou des esprits du règne animal ou végétal ayant été brusquement et prématurément arrachés à I'existence, encore trop désireux de participer aux flux et reflux de la vie pour se résoudre à aller trouver du repos au pays de l'origine. 
Ces âmes ou ces esprits, ayant été brisés dans leur élan, demeurent chargés de propensions à canaliser les événements conformément aux orientations qu'ils avaient prises. Ils identifient des traces encore agissantes d'un passé récent imputables à des créatures empêchées désormais de se manifester directement, ne parvenant à s'affirmer qu'en induisant des vivants à partager leurs désirs, en les tracassant à la façon dont un enfant n'a de cesse d'importuner sa mère pour attirer l'attention sur lui ou en se mêlant d'une autre façon quelconque aux péripéties de leur vie. Les noeuds d'énergie orientée qu'ils représentent embrouillent ou déforment dans une direction déjà poursuivie les voies de précipitation des événements vers le monde visible.

La plupart des divinités les utilisent aussi bien pour tourmenter les personnes qui ne satisfont pas à leurs volontés que pour tourner le sort à l'avantage de celles qui leur plaisent en s'opposant, le cas échéant, à l'action en sens contraire d'autres agents immatériels. Elles les mettent parfois aussi à contribution pour provoquer une transe de possession ou pour transformer en cours d'initiation la personnalité des adorateurs qu'elles se sont choisis. L'énergie subtile inévitablement dépensée au cours de tels travaux doit périodiquement être restituée à leurs subordonnés par les émanations du sang des victimes sacrificielles déversé sur ou devant le ou les objets servant à ces derniers d'habitacle temporaire ou permanent.

En tant que telles les divinités n'ont aucun besoin d'être gratifiées de sang. Cependant, les objets en question, du fait qu'ils servent à identifier les moyens spirituels ou organes d'action dont elles disposent, leur tiennent couramment lieu d'autels. Périodiquement lavés et remis en état en tant que creusets de reconstitution d'une puissance permettant une relative mâttrise des phénomènes, ils n'en restent pas moins intégrés au domaine purement religieux qui est celui des relations de l'homme avec des entités autonomes dont il dépend pour l'essentiel et devant lesquelles il s'incline.

Cependant les esprits des créatures dont la vie fut trop brusquement interrompue ne sont pas tous accueillis au service des divinités. Certains viennent directement semer le trouble dans une famille jusqu'à s'y faire accorder I'hospitalité ou se laissent volontiers 
utiliser par des particuliers ayant réussi à contracter alliance avec eux. On leur installe quelque part un habitacle analogue, périodiquement réaménagé et lui aussi arrosé d'un peu de sang pour les satisfaire. Il est remarquable que les Evhé ne le confondent pas davantage avec un support daction magique du type de ceux qu'ils utilisent couramment par ailleurs (voir plus bas, pp. 275-277) pour prendre ascendance sur des esprits. Dans les limites, toujours assez floues, de l'accord ayant été passé avec eux, les agents invisibles y ayant pris résidence demeurent entièrement libres de leurs actions et ne font pression que comme ils l'entendent sur le cours des événements.

\subsection{Possibilités d'interventions humaines au niveau d 'un tel processus}

L'homme n'est pas entièrement dépourvu devant les mauvais coups du sort ou de la fatalité. En se contentant d'intervenir à proximité immédiate de lui-même il peut compléter la protection qui lui est offerte par les divinités, les ancêtres et les esprits indépendants au moyen d'une très riche panoplie d'astuces psychiques et d'actes de magie... comme si, plutôt que d'arrêter la pluie à sa source ou de déclencher un vent la repoussant un peu plus loin, il trouvait moins compliqué de drainer correctement les eaux ou de se fabriquer un parapluie pour l'ouvrir en cas de besoin.

\subsubsection{L'esquive d'événements inévitables}

Il n'y a guère d'autre moyen de se protéger d'événements contenus en germe dans la portion de nature prise en charge par l'âme que de livrer en pâture aux représentants supposés de la fatalité (appelés gbetsi, "voix ancienne") des réalisations symboliques ne portant préjudice à personne, de ce qu'ils arrivent exiger ou ne manqueront pas tôt ou tard d'exiger. Créatures de l'imagination, ils se contentent en effet très volontiers de satisfactions imaginaires. 
Viennent-ils imposer à un individu l'expérience malheureuse que lui vaut son choix prénatal, on détourne leur attention sur des figurines d'argile fraîche très sommairement façonnées qui le représentent en l'état non cuit et inachevé qui est celui attribué aux créatures du monde de l'origine. Pour mieux endormir leur vigilance, leur donner l'illusion qu'ils trouvent bien là ce qu'ils sont venus chercher ou les convaincre d'accepter le substitut qu'on leur présente, on ajoute au même endroit divers ingrédients et semblants d'offrandes : des bouts de chiffons représentant des pagnes, des moitiés de papaye évidées les calebasses de boisson, quelques cauris d'importantes sommes d'argent, et des miniatures sans valeur des objets réels.

En cas de malheurs approchant à grandes pas ou de sanctions expressément décidées par les ancêtres à la suite de violations de la loi morale, on détourne pareillement l'événement estimé être inévitable en le dirigeant, de façon plus frappante encore, sur des victimes réelles : ce sont le plus souvent des quadrupèdes que $l^{\prime}$ on met à mort avec une cruauté absolument incompatible avec un acte sacrificiel et dont on se débarrasse ensuite en brousse ou au dépotoir en se gardant bien d'en consommer les chairs.

Quelqu'un est-il empêché de mener sa vie conjugale conformément à l'idéal qu'il s'est constitué au pays de l'origine, on lui fait adopter une statuette en bois de sexe opposé au sien, ou bien même on lui choisit dans le village une autre personne de sa génération inépousable par lui, avec laquelle il est invité à entretenir des relations matrimoniales purement symboliques actualisant, d'une façon qui ne vient pas interférer avec son existence réelle, un mode d'expérience de la vie qu'il était jusqu'alors empêché d'éprouver. Le but visé n'est plus ici de leurrer une redoutable entité de l'au-delà par un objet de forme aussi imprécise qu'elle, mais de contenter une créature de chair et d'os au moyen d'une représentation pareillement semblable à elle, cette fois vivante ou taillée avec précision dans un matériau dur. 
De même, un jeune enfant souffre-t-il de la perte de son jumeau, celui-ci est représenté auprès de lui par une statuette. en bois dont la mère prend symboliquement soin de la même façon que de lui pour que, manquant moins de sa présence, il soit moins tenté de le rejoindre dans la mort.

Subsiste-t-il dans l'imaginaire collectif, au niveau du conte populaire transformé en conte divinatoire, une croyance en l'efficacité de certaines conduites pour écarter un risque de mésaventure, alors une sorte de concrétion matérielle du conte en question, appelée vosa ("attacher le vol"), le réactualisant puissamment dans l'esprit de qui s'y réfère, est constituée pour dissuader les agents qui acheminent secrètement le mal de poursuivre plus avant.

Tous ces moyens de donner satisfaction, sans devoir soi-même en souffrir, à des instances psychiques avec lesquelles on ne transige pas et qui précipitent vers le monde sensible des événements fâcheux réussissent à proportion de la foi que les participants ont en eux et de l'énergie vitale qu'ils y investissent en $y$ ayant recours.

\subsubsection{Le détournement du sort à son avantage}

Dans la mesure où le hasard qui participe à la fixation du devenir $\mathrm{du}$ monde se laisse invisiblement manipuler, l'homme peut apprendre à attirer directement à lui le maximum de chances, de richesses, de renommée, de pouvoir social, et de naissances... ou à précipiter inversement sur d'autres que lui le maximum de malchances, en provoquant notamment le malheur ou la destruction de ses rivaux.

\section{a) Les médecines magiques}

Tout phénomène est jugé favorisé ou contrarié par l'atmosphère où baignent les esprits (incarnés ou désincarnés) qui contribuent à l'engendrer ou à l'entretenir. Certaines ambiances facilitent l'actualisation de tel ou tel tandis que d'autres la contrarient. Il y en $a$, en particulier, qui attirent des esprits errants avides de susciter chez les vivants les émotions qui leur plaisent, tandis 
que d'autres, à la façon d'odeurs nauséabondes, agissent sur eux comme un repoussoir.

De telles ambiances peuvent être créées par de savantes combinaisons de souffles (gbogbo) émanant de l'extrémité des végétaux, parfois de leurs racines mais essentiellement de leurs feuilles. On distingue à cet effet des plantes chaudes (dzodzo) ou excitantes et des plantes fraîches (fafa) ou calmantes. Ou bien on les plonge dans l'eau pour préparer un amatsi ("eau de plantes magicomédicinales") dont on imprègne un sujet ou un autel de divinité si l'on veut augmenter d'ardeur des esprits qui y siègent ou leur inculquer la paix; ou bien on les soumet à l'action du feu en les calcinant dans une terrine pour les réduire en une poudre noire appelée eti si l'on veut repousser loin du sujet, de son habitation ou d'un autel de divinité les esprits qui le fréquentent ou réduire seulement l'excitation de ceux qu'on ne souhaite surtout pas. en éloigner.

b) Les charmes

On passe insensiblement des "médicines" aux charmes (bo). Les géomanciens représentent d'ailleurs ces derniers par le même petit symbole (une gousse de poivre de Guinée) que les pots d'amatsi et les poudres eti. En effet une "médicine" exerce déjà un certain "charme" sur les esprits du patient ou sur ceux qui le fréquentent familièrement et le protègent. Elle perd toute efficacité sans le consentement ou l'appui de ces derniers.

Le nom de charme (bo) est néanmoins réservé à une mâttrise plus vigoureuse d'invisibles agents de propagation de phénomènes dont on veut être préservé, dont on veut priver autrui ou que l'on veut au contraire précipiter sur autrui. Il évoque un attachement (sasa) de ces agents avec les solides cordelettes que l'on fabrique avec l'écorce d'un arbre appelé bo-ti ("I'arbre bo" ou "l'arbre du bo").

Une telle maltrise, à laquelle parviennent des individus que I'on nomme boto (propriétaires de bo) ou dzoto (propriétaires de "feu"), ne se borne pas à la création, au moyen de plantes, d'une atmosphère appropriée. Elle requiert la prononciation de paroles 
magiques contraignantes ${ }^{1}$ et la minutieuse exécution d'un rite de fabrication ou de mise en oeuvre. Ce rite met généralement en jeu des instruments ou divers autres ingrédients qui peuvent être détruits à chaque opération, mais dont certains, pour plus de commodité, sont parfois conservés et tiennent alors lieu de supports matériels permanents d'actes magiques qu'ils finissent par suffire à identifier.

On notera que les paroles requises ne sont pas désignées par le même mot (nyà) que les paroles ordinaires, mais par celui de gbesa qui signifie "nouage" ou "attachement" de la voix (gbe). Gbe ne se rapporte pas au contenu d'un énoncé mais à la manière de parler et au langage employé, c'est-à-dire à ce qui le supporte et lui demeure antérieur, se posant en inévitable intermédiaire entre l'intention de dire quelque chose et son affirmation effective. Les gbesa n'apparaissent donc pas destinés à produire du sens mais à affirmer vigoureusement une volonté de réaliser une intention. Elles doivent de plus être récitées bribe par bribe en crachant sur les plantes et les autres ingrédients rassemblés des grains croqués de poivre de Guinée (ou exceptionnellement de mil pour agir dans le bon sens sur des esprits de divinités).

Présentée par le mythe comme un élément de confirmation par Dieu ou par la Mère universelle de tout ce qui est fabriqué à l'état de germe au seuil du monde de l'origine, la salive symbolise le don du souffle chaud et humide dont il est nécessaire d'enrichir

1 Tout en évoquant une puissance spécialisée, de telles paroles l'inclinent à agir dans le sens voulu. En voici deux brefs exemples: a) pour exercer un charme destiné à repousser la lèpre : "Dzadzagliga ! wo nonlodzali, medhi kpovi le be kpovi ne nyo. Aphedovhu na dze agbagbà naxè" (Quelque chose d'énorme! Les plaies de la lèpre. Il ne ressemble pas au petit léopard mais se dit petit léopard. L'aphedo (le lignage) est ouvert, fais tout pour le fermer) ;

b) pour installer sous forme d!Apheli un bo-vodu protecteur de la maison : "Eku le aphe. Trovô ne ku le aphe ! Adrigba ! Tome kpe be ye dhen dhi na vuvo" (Il est mort à la maison. Que le mauvais tro meure à la maison! Qu'il glisse (et tombe) ! La pierre de la rivièré dit qu'elle se moque du froid).

Les devises des ancêtres ont elles aussi un pouvoir contraignant, mais seulement celui de les obliger à se présenter dans une cour ou sur un autel pour y écouter une prière; ayant écoutée celle-ci, ils restent libres de leurs décisions. 
toute décision créatrice pour que l'ordre de l'exécuter soit transmis au monde. En étant projetée de la sorte avec les paroles, elle nous indique que le charme opère, non pas au niveau des choses ou expressions achevées, mais à celui où les esprits se trouvent emportés dans leurs actions par le "souffle" qui les inspire.

1.5.3 Subjugation d'esprits désincarnés au moyen de charmes

Contrairement aux simples "médecines" dont l'action reste locale, un charme est censé pouvoir agir à distance. On est tenté d'en conclure que son efficacité dépend de l'intervention d'esprits extérieurs à ceux de son utilisateur, allant sur sa requête exécuter diverses tâches. Mes meilleurs informateurs m'ont cependant tous affirmé que les résultats qu'on en obtenait ne dépendaient nullement du bon vouloir d'une divinité, d'un ancêtre ou d'un esprit errant, d'origine humaine ou extra-humaine, mais étaient produits automatiquement à proportion de la conviction et de la rigueur rituelle déployées pour l'exercer. Ou bien il influence directement les esprits de personnes vivantes, ou bien il influence des esprits vitaux de défunts n'ayant pu encore être accueillis au pays de leurs ancêtres, ou encore divers esprits de la nature. Dans de nombreux cas il permet tout simplement de repousser ou de calmer de tels esprits désincarnés pour ne plus en être importuné. Cependant il sert parfois à capturer et domestiquer certains d'entre eux pour profiter à volonté de leurs capacités de vigilance, de connaissance ou d'agression.

Un charme permanent situé dans une habitation ou aux portes d'une habitation ou d'un village devient souvent ainsi le point d'attache d'un esprit errant ou d'une bande d'esprits errants assimilable à un démon. Tel est notamment le cas de ceux que l'on appelle bo-vodu ("charme-vodu" ou "vodu de charme"). Habituellement installés en enfonçant dans le sol, ou sous une idole ou une élévation de terre symboliquement décorée, une poterie remplie de feuilles appropriées et de divers autres ingrédients, ils contrarient l'action de mauvais charmes prenant appui sur une même puissance que la 
leur, retiennent éventuellement auprès d'eux des esprits charmés mal intentionnés, ou deviennent surtout fréquentés par des esprits auxquels il plaisent, acceptant en contrepartie de remplir telle ou telle tâche de pretection. Les plus répandus sont les Apheli et les Legba. Pour la construction des plus importants de ces derniers on $n$ 'hésitait pas autrefois à fabriquer tout spécialement des "mauvais morts" en enterrant par-dessous des esclaves dont les esprits étaient contraints d'y demeurer attachés.

De méchants esprits, secrètement capturés de la sorte par des individus, demeurent à leur disposition pour les défendre ou pour attaquer leurs ennemis, à la façon de bêtes fauves sous l'autorité de leur dompteur. Leur entretien es coûteux et nécessite la plus extrême vigilance. Sur un seul geste qui les irrite, contraire à leurs interdits, ils n'hésitent pas à attaquer leur propriétaire. Au cas oùils ne sont pas suffisamment abreuvés de sang pour récupérer l'énergie vitale qu'ils dépensent en exécutant ce que celui-ci leur ordonne, ils n'hésitent pas à le faire périr, à absorber sa vitalité ou celle d'un membre de sa famille.

Cependant des esprits moins redoutables, captés par un charme dont l'usage n'a pas été contraire à la loi morale, peuvent être reconnus par les ancêtres comme un bon moyen d'intervention dans les affaires du monde au bénéfice de leurs descendants. Après la mort de celui qu'ils ont servi, ils se transforment, en association avec lui, en un organe d'action d'une nouvelle divinité identifiée par l'instrument ayant servi à les subjuguer.

En définitive il nous faut établir une claire distinction entre la propre puissance des plantes (la facilitation de l'activité de certains esprits par le souffle qui slen dégage), celle du charme (permettant une projection de la volonté de son utilisateur avec l'appui complémentaire d'objets symboliques, de paroles crachées et de gestes rituels), celle d'esprits désincarnés éventuellement contraints à l'action par l'exercice du charme, enfin celles d'ancêtres appuyant ou contrôlant parfois 1 'activité de tels esprits désincarnés. 


\subsubsection{Le cas des vodu-médicaments (atikè-vodu)}

Sans attirer nécessairement à lui des esprits et donc se transformer en habitacle de démon ou de divinité, un charme permanent, matérialisé d'une manière analogue à celle d'un bo-vodu, destiné à guérir des maladies ou à écarter divers malheurs en mâtrisant de mauvais esprits (notamment ceux de sorciers) donne parfois lieu à une association d'adeptes dont les rassemblements, ̀̀ l'occasion des cérémonies de reconstitution de sa puissance, rappellent étrangement ceux que suscite un véritable culte. On le qualifie plus précisément d'atikè-vodu ("médicament-vodu" ou "vodu de médicament").

La sommation autour de lui des attentes d'un nombre important de personnes, anxieuses de recouvrer la santé ou la paix, a pour effet d'augmenter considérablement la puissance de l'"atmosphère" qui le caractérise, au point que certaines d'entre elles succombent littéralement sous le poids de la charge psychique qu'elles sont amenées à supporter. Il semblerait que, tout en retrouvant auprès des autres adhérents et sympathisants le sens de la communauté humaine, elles déposent alors à l'extérieur d'elles-mêmes les principaux facteurs de leurs blocages et parviennent, en adoptant certains comportements outranciers théâtralisés, à épuiser une grande part de l'énergie qui y demeurait attachée.

Avec ou sans la présence d'esprits errants désincarnés attirés sur les lieux, il arrive qu'en collaboration avec des âmes d'ancêtres établissant pour elles une connexion avec le monde de l'origine (parfois seulement en collaboration avec leur ancêtre tutélaire) et mettant à profit l'écrasement de leurs propres facultés mentales, elles soient sujettes à des transes de possession bien plus spectaculaires que celles qui se produisent au cours des cérémonies du culte des grands vodu ou sont provoquées en privé à des fins divinatoires. Elles se mettent à prophétiser, à révéler des choses cachées, à dénoncer de mauvaises conduites, à indiquer les exigences de charmes permanents ou d'esprits, ou à transmettre directement à la foule la parole des ancêtres ou des divinités. 


\section{LA DIFFICULTE DיY DISTINGUER DES FETICHES}

Parmi les différentes puissances ou les différents moyens auxquels les Evhé font appel (Se, tro, vodu, nolli, gbetsi, vosa, bo) pour tenter d'échapper à la nécessité naturelle, à la fatalité ou à de néfastes influences, y en a-t-il que nous puissions convenir de désigner utilement et sans ambiguïté par le terme de fétiche ?

Plusieurs figurations entretenues par un sujet demeurant persuadé d'en dépendre pour son bonheur pourraient prétendre à ce titre :

- substituts matériels de personnes vivantes (tels les silhouettes ou les simples têtes en argile fraîche servant à renvoyer de mauvais gbetsi ou entrant dans la composition de nombreux rosa) abandonnés à d'invisibles propagateurs de maladies et de malheurs délégués sur terre depuis le monde de l'origine, dans l'espoir qu'ils s'en contenteront et cesseront d'exiger la réalisation effective des phénomènes qu'ils s'entêtent à imposer ;

- substituts matériels durables et plus élaborés de personnages disparus (tels les statuettes de jumeaux) destinés à préserver les personnes qui leur étaient attachées de la tentation de les suivre dans l'autre monde;

- enfin et surtout, substituts matériels de personnages déclarés avoir été fréquentés au pays mythique de l'origine avant d'être envoyés prendre naissance (tels les statuettes de conjoints de l'audelà), permettant à un sujet de compenser les contrariétés de la vie réelle, à la façon dont un enfant prend pour compagnon une poupée, par des conduites symboliques conformes à des idéaux indéracinablement implantés dans les profondeurs de sa nature.

Il ne viendrait cependant à l'idée d'aucun Evhé de déifier de telles figurations ou de les supposer chargées d'une puissance magique qu'il conviendrait périodiquement de reconstituer. Par ailleurs, alors qu'on attribue habituellement aux fétiches un caractère dangereux et ambigu, aussi propice à l'attaque qu'à la défense, sans pitié pour qui en enfreint les interdits, pouvant indifféremment oeuvrer pour le bien ou pour le mal, elles sont toujours rassurantes pour celui qui en fait usage et n'offensent jamais personne en contrepartie. L'atmosphère qui en entoure la consécration et le 
traitement $n^{\prime}$ a rien de comparable avec celle de dissimulation et de lutte féroce, inspiratrice d'une terreur analogue à celle d'être empoisonné ou frappé à la moindre chute de vigilance, où opèrent ceux que l'on nomme le plus volontiers féticheurs.

Assurément le terme ne convient guère aux génies (Se), aux divinités (tró) et aux ancêtres (noli) considérés en eux-mêmes, mais pourrait être réservé aux autels, reposoirs, sièges ou habitacles divers en la matière desquels ils semblent s'incarner : généralement des éléments de la nature ou des objets dont l'aspect, la composition ou l'histoire rappellent aux hommes l'existence de divers principes de l'univers, d'entités protectrices ou de points de repère moraux. Il ne s'agit pas là en effet de simples dénotations de réalités invisibles, mais de symboles avec lesquels il est nécessaire de composer, représentant, conformément à une conception originale du monde, des facteurs objectivement fixés d'organisation ou de déformation de l'espace des comportements. Un fétiche se définirait alors comme un élément structurel non négligeable de cet espace et tout un chacun, qu'il en ait conscience ou non, et quelle que soit sa culture, prendrait nécessairement appui sur des fétiches. La notion s'en trouverait abusivement étendue à toute espèce de monument ou de souvenir.

Le terme pourrait pareillement être appliqué aux habitacles spécifiques d'âmes de "mauvais morts" (mauvais noli) ou d'esprits de la nature livrés à eux-mêmes ou ayant été acceptés au service des aivinités et servant alors à ces dernières d'organes d'intervention dans le monde des hommes. Compte tenu de leur irrésistible envie d'être occupés en se mêlant toujours de quelque chose, à la différence des entités qui gardent pied ou ont repris pied dans le monde de l'origine, ces âmes ou ces esprits s'avèrent avant tout causes de tracas et d'aliénation. Provocateurs de résonances, d'accélérations et d'emballements, ils représentent des facteurs de mobilité au sein d'une structure caractérisée par son inertie. Les uns s'emploient à la contester ou même à l'ébranler jusque dans ses fondements. Les autres au contraire contribuent à en assurer la conservation. Dans l'espace des comportements un fétiche correspondrait alors plutôt à une poche d'énergie ou à un certain courant d'énergie 
contre lequel l'homme doit lutter pour progresser, mais dont il peut apprendre à tirer parti comme un voilier profite du vent. La notion en serait cette fois surétendue à toute induction par les traces concrètes ou verbales d'initiatives antérieures, comme à tout moyen d'objectivation d'une angoisse.

Outre pareille extension, ce qui dissuade surtout, dans l'un comme dans l'autre cas, de parler de fétiche est la totale incapacité des vivants à manipuler eux-mêmes la puissance ainsi localisée. Ou bien elle est détenue par une entité indépendante dont ils se méfient des caprices, de la roublardise ou même de la méchanceté, et avec laquelle ils traitent donc avec une grande prudence, ou bien elle est gouvernée par une divinité qui en a fait sa propre chose et qu'ils sont réduits à supplier humblement de. les aider. Or nous sommes enclins à penser que, par opposition à un prêtre ayant été "saisi" par une divinité ou à tout autre intermédiaire entre les hommes et les invisibles puissances qui l'environnent, l'homme méritant le titre de féticheur négocie moins avec des esprits qu'il ne réussit à les dominer, et que, contrairement aux actes d'adoration, d'adulation ou de prière, les actes fétichistes sont de nature à produire immédiatement et quasi-automatiquement leurs effets.

c'est, semble-t-il, en se tournant vers les bo et les dzo que l'on touche de plus près au domaine du fétichisme, car on leur prête un effet contraignant instantané sur des esprits, que ce soit à des fins attractives, défensives ou agressives. De plus leurs détenteurs (boto ou dzoto) gardent sur eux, sauf négligence ou erreur technique de leur part, un total contrôle ${ }^{2}$. Cependant, alors que nous avons été habitués à associer si étroitement la notion de fétiche à celle d'objet, au point que l'on parle couramment d'objet-fétiche, un bo n'est pas toujours matériellement repérable.

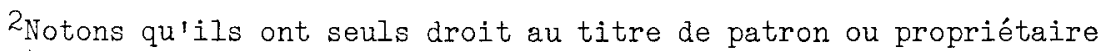
(to) des objets puissants dont ils disposent. Les responsables du culte (huno, bokono, tronunola ou tronua) sont désignés à l'aide des suffixês no (cêlui qui se tient $\overline{\grave{a}}$ côté de ou au service de), nunola (celui qui se tient à la bouche de) ou nua (bouche de). Les autres prêtres et initiés (trosi et vodusi) sont désignés par le suffixe si (épouse de) caractérisant la soumission quasi-sexuelle de leur personne à leur divinité. 
Il se limite soluvent à la connaissance d'une recette magique mettant en jeu des ingrédients, principalement végétaux, d'indispensables paroles magiques (gbesa), parfois inintelligibles, et un rituel de préparation de quelque chose.

La puissance de nombreux bo est réactualisée à chaque occasion indépendamment de ses actualisations antérieures. Il arrive cependant que, parmi les ingrédients solides non périssables à utiliser, certains soient de facture suffisamment laborieuse pour qu'il soit jugé préférable de les conserver en l'état d'une fois sur l'autre. A de tels objets mobiles, soigneusement entreposés au secret dans les cases de leurs propriétaires, convient-il a'appliquer le terme de fétiche ? On hésite à s'y résoudre dans la mesure où ils sont considérés comme de simples accessoires dont la connaissance du mode d'emploi est bien plus importante que leur possession. Qui sait comment procéder saura toujours en effet réunir les éléments nécessaires au rite ou à la préparation envisagée, mais qui n'est mis en possession que d'un ou plusieurs de ces éléments n'est guère plus avancé s'il ne sait correctement en faire usage.

Voici par exemple deux statuettes, mâle et femelle, dont un certain mode de traitement est destiné à réconcilier ou au contraire à brouiller deux époux ou deux amants. Le nom de bo ne leur est pas exclusivement réservé mais désigne la connaissance de tout ce qu'il faut faire avec elles pour obtenir le résultat escompté. Pour autant qu'elles en constituent la partie visible, elles identifient bien entendu le bo en question, mais celui-ci ne s'y résume en aucune manière.

Sous réserve d'une telle conservation d'éléments réutilisables une ou plusieurs fois, les bo destinés à produire à distance un effet instantané ne sont souvent pas concrétisés en permanence. On peut aller en acquérir, auprès de quelqu'un, c'est-à-dire recevoìr auprès de lui le pouvoir d'appliquer avec efficacité la recette correspondante, et revenir chez soi les mains vides. Cependant il en va différemment avec ceux dont l'effectuation aboutit à concentrer pour un temps dans un produit, un objet ou un lieu précis, de bonnes ou de mauvaises influences. Ou bien le résultat concret de l'opération est périssable (cas d'une préparation offensive conçue pour ne pas 
être découverte, à disperser ou à enterrer dans la cour de son ennemi), ou bien il ne l'est pas ou ne l'est partiellement pas et, plutôt que d'être entièrement refait à chaque fois, est seulement réaménagé ou rechargé. Est-ce à de tels aboutissants de l'action magique qu'il conviendrait d'appliquer plutôt le terme de fétiche?

Qu'il s'agisse d'une idole, d'un objet parfois réduit à une simple pierre, ou d'un curieux agrégat de substances..., leur puissance est en effet périodiquement reconstituée au moyen de rites comportant des versements de sang. Ainsi semblent-ils, aux yeux d'observateurs inavertis, être l'objet de sacrifices identiques à ceux rendus sur des autels d'ancêtres ou de divinités, et peuvent-ils être dénoncés par eux comme des dieux factices ou comme des objets pris pour des dieux. En réalité pourtant, à la différence d'objets matériels de valeur ainsi que de sanctuaires de divinités dont on attend qu'elles se reportent d'elles-mêmes sur quelqu'un, les bo sont uniquement transmissibles du vivant de leur possesseur (certes dans bien des cas de père en fils) ${ }^{3}$. Ils ne: s'héritent pas. Si celui ou celle qui en a acquis ou installé un meurt avec ses secrets, les objets correspondants sont laissés à l'abandon, détruits par le feu ou enterrés..., selon les préférences de sa famille et les indications d'un devin.

Il arrive souvent que les influences recherchées soient concentrées à même les ingrédients nécessaires à l'effectuation du bo. Il y a alors coìncidence entre un ou plusieurs accessoires non périssables employés et le résultat concret de l'opération. Tel est précisément le cas des bo-vodu. Arrêtons-nous y un instant car il nous démontre bien l'immatérialité de la source de puissance du genre d'objets considérés et le fait qu'elle ne leur est pas jugée inhérente, mais laborieusement introduite en eux.

Ils doivent en effet l'essentiel de leurs vertus à une composition végétale parfois insérée dans une statuette ou un sachet, mais généralement enterrée dans une poterie ou enfouie à la base de

3 Exceptionnellement un homme ayant péri brusquement, avant de communiquer ses secrets au fils ou au neveu dont il voulait faire son successeur, pourra bientôt les lui inspirer en rêve. 
l'idole, de la pierre ou du monticule porteur de symboles qui les identifie. Cette composition ne tarde pas à pourrir et devient donc matériellement inexistante. Curieusement, elle n'est pourtant jamais renouvelée. Elle constitue seulement l'amorce ou le foyer de fixation d'une sorte d'ambiance analogue à celle qui, une fois créée dans un café à l'aide d'artifices d'éclairage, de décorations et d'amabilités, s'entretient ensuite indépendamment par la seule fréquentation qui a commencé d'y être assurée. Il est clair qu'aucun objet en tant que tel n'est pris ici pour fétiche : ni les plantes dont ensuite on ne se soucie guère, ni leur enrobage matériel durable qui serait resté sans elles une simple chose. Cet enrobage n'est pas puissant par lui-même ; il est investi de puissance mais n'est pas pour autant investi par des esprits car il va permettre au contraire d'agir comme on le souhaite sur les esprits.

Nous le constatons ici, une puissance ayant été fabriquée à l'aide de plantes peut être emmagasinée sur un support matériel. Du fait qu'un même ingrédient ou un même groupe d'ingrédients est souvent nécessaire à l'effectuation de plusieurs bo possédés par un même homme, l'idée vient aisément à ce dernier de fabriquer et de sauvegarder à proximité de lui la puissance correspondante qui en représente le facteur commun. L'effectuation de ses bo n'exige plus dès lors que quelques rites complémentaires ressemblant au prélèvement d'une puissance spécifique (sous forme de produits, d'amulettes ou de sortes de bénédictions...) dans une masse de puissance accumulée sur un objet éminemment précieux pouvant prétendre aussi à l'appellation de fétiche. Périodiquement remis en état et abreuvé de sang, il donne encore plus vivement l'impression de recevoir un culte abusif alors que le magicien qui s'en sert ne le traite qu'en réceptacle d'énergies subtiles à mobiliser par des paroles et des actes adéquats.

Ayant le pouvoir de contraindre aussi bien les esprits désincarnés que les esprits de personnes vivantes, les bo servent parfois, comme on I'a vu, à capturer et domestiquer, pour profiter de leurs surprenantes capacités, des esprits de la nature ou des âmes errantes de "mauvais morts". Il suffit que des ancêtres aient accepté de couvrir de leur autorité 1 'activité d'un magicien ayant réussi à en subjuguer ou les ayant reçus en héritage, pour que l'instrument ou le signale- 
ment matériel du charme ayant été employé soit élevé, du fait de leur honorable présence, au rang d'autel, non plus de simple démon (bon ou mauvais), mais de nouvelle divinité s'en servant comme moyen d'intervention dans le monde.

De telles pratiques permettent aux hommes de rénover constamment, en les adaptant à leurs besoins, la population des entités invisibles auxquelles ils jugent bon d'avoir recours. Très répandues en Afrique Noire, elles remontent aux sources mêmes de la tradition hermétique. En effet, lisons-nous dans 1'"Asclépius" (XIII, 37-38), traité remontant au début du 4 ème siècle de notre ère, "Nos premiers ancêtres... inventèrent l'art de faire des dieux... Ils y attachèrent une vertu appropriée qu'ils tiraient de la nature matérielle ; et, mêlant cette vertu à la substance des statues, comme ils ne pouvaient créer proprement des âmes, après avoir évoqué des âmes de démons ou d'anges, ils les introduisirent dans leurs idoles par des rites saints et divins, en sorte que ces idoles eussent le pouvoir de faire du bien et du mal...

"- De ces dieux qu'on nomme terrestres, ô Trismégiste, de quelle sorte est la propriété ?

" - Elle résulte, Asclépius, d'une composition d'herbes, de pierres et d'aromates qui contiennent en eux-mêmes une vertu occulte d'efficacité divine... Voilà comment 1 'homme fabrique des dieux."

Les habitacles matériels d'esprits ainsi asservis méritent probablement le mieux l'appellation de fétiche avec toute l'ambiguité du terme : chose fabriquée ou artificielle, chose puissante mais dangereuse car pouvant basculer aussi bien dans le registre démoniaque que dans le registre divin. Quand il arrive à la puissance qui y siège de se transformer en organe ou en efficace d'une divinité, la divinité parât finalement mettre cette puissance à la disposition de l'homme alors qu'il est revenu à ce dernier de la fabriquer et qu'il continue seul, au moyen d'opérations rituelles lui ayant été inspirées ou enseignées, à pouvoir la mettre en oeuvre. Difficilement pensable dans le cadre des théologies auxquelles nous sommes accoutumés, cette situation ne suffit-elle pas à justifier la défense et l'illustration de systèmes à bon droit qualifiables de "fétichistes"? L'application du terme aux attracteurs ou aux lieux d'atta- 
che d'esprits offrirait de plus l'avantage de correspondre à l'emploi qu'en fit Tylor et de nombreux autres anthropologues. "Beaucoup de peuples primitifs, écrit $\mathrm{H}$. Webster ("La magie dans les sociétés primitives", Paris, Payot, 1952, p.140), possèdent... des objets matériels, inanimés, dont la puissance occulte est due à leur possession temporaire ou permanente par des êtres spirituels : ce sont les fétiches. L'esprit du fétiche n'est pas son âme, son essence vitale, c'est un esprit qui s'est laissé attirer de plein gré ou qui a été acculé par l'homme dans l'objet et s'y est incorporé".

Cependant, les "médicaments-vodu" antérieurement évoqués, qui se font immoler de grands animaux et suscitent fréquemment la possession de leurs fidèles, ne représentent-ils pas encore mieux, alors que dans bien des cas aucun esprit désincarné ne s'y trouve attaché, les objets bizarres ou horrifiants que les indigènes de la "Côte de Guinée", à la plus vive indignation des Européens, semblaient entourer des mêmes égards que leurs dieux en s'en souciant surtout bien davantage que de leur propre Dieu suprême? Ils peuvent en effet être reconnus bénéfiques par tout un quartier ou un village et donnent lieu à de très importantes festivités analogues à celles de cérémonies religieuses, organisées par commodité à la même époque que ces dernières durant les semaines d'abondance consécutives aux récoltes. En faveur de cette option on notera que plusieurs objetsfétiches d'origine sahélienne introduits dans le pays par les travailleurs migrants du Ghana, et dont certains ressemblent de près à des boli bambara ou des yapèrè minyanka, se trouvent classés parmi eux.

Décider de limiter le terme fétiche aux constituants matériels des bo ayant permis l'incorporation d'esprits dans des objets pour fabriquer de nouveaux daïmons (mot couvrant un ensemble d'entités dont l'appartenance à la classe des dieux ou des démons n'est pas définie) laisse donc subsister quelques difficultés. Ceci nous amène à nous demander si le fait de le conserver, même après en avoir choisi une définition précise, ne présente pas finalement plus d'inconvénients que d'avantages.

D'une part l'exclusion du champ sémantique correspondant d'une grande proportion de ce que l'on trouve de ci de là désigné ainsi risquerait pendant longtemps d'induire plus d'une erreur. 
D'autre part, qu'on le veuille ou non, trop d'idées fausses relatives aux religions des "primitifs" lui restent attachées. Il laisse notamment croire que les grands enfants que certains ressortissants des pays sous-développés demeureraient accorderaient des pouvoirs et rendraient un culte à des choses. Or nous avons pu constater que ces dernières ne sont considérées par les Evhé qui les manipulent, et parfois les thésaurisent, que comme des supports ou des contenants d'une puissance immatérielle s'y trouvant spontanément accumulée ou y ayant été introduite par des experts.

Mais surtout il n'apparâ̂t pas de nature à jeter quelque lumière sur l'ensemble des réalités à propos desquelles son usage prête à discussion. Au lieu de nous laisser obnubiler par lui, nous ferions sans doute mieux de nous référer à d'autres notions.

\section{NOTIONS CONVENANT MIEUX A LA PRESENTATION COMME A L'ANALYSE DES FAITS}

Tout bien considéré, les notions evhé de puissance spirituelle (gbogbo), de force opérante ou de charme (bo), de milieu énergétique nous tenant séparé de notre point de rattachement à la nature universelle et d'esprit (luvho) s'y trouvant plongé et nourri, apparaissent plus pertinentes, pour l'analyse des croyances et des phénomènes considérés, que celle de f'étiche que nous avons essayé d'y appliquer. Nous allons tenter d'en préciser ci-dessous le contenu en un langage rationnel.

\subsection{Notion de puissance spirituelle}

Les informateurs les plus compétents : mâtres géomanciens, (bokono), dignitaires des cultes vodu (huno) ou féticheurs (boto ou dzoto) renommés, nous enseignent tous, sans ambiguìté, que le support essentiel d'un acte de magie n'est pas un objet (notamment un objet "fétichisé", pris pour catalyseur ou pour témoin d'une réalisation imaginaire de propensions refoulées), mais un gbogbo, 
c'est-à-dire un "souffle" en affinité éventuelle avec la forme, l'usage et l'imprégnation antérieure de certains objets. Il nous signifient ainsi qu'il n'appartient pas au domaine des réalités ou des représentations objectives, mais à celui de l'invisible étayage de celles-ci, intermédiaire entre celles-ci et le fondement par rapport auquel elles prennent un sens. La substance dont il se compose est celle d'un impalpable milieu où baignent tous les esprits et dont se nourrissent tous les esprits. Elle est propre, de ce fait, à assurer la continuité, exigée par notre sens commun de la causalité, de tout transfert d'influence entre deux ou plusieurs agents d'exécution de la manifestation divine.

Le gbogbo en question, précisent-ils, émane spontanément sans intervention complémentaire de qui que ce soit, des matières végétales (feuilles et parfois aussi racines) qui ont été rassemblées. Toute sa puissance (nuse) provient des plantes. Cependant cette affirmation doit être tempérée par les faits suivants démontrant que ce sont moins les plantes elles-mêmes qui agissent que la façon dont elles sont percues par leurs utilisateurs en fonction d'une théorie communément admise.

- Les autels-fétiches des mêmes vodu ou bo-vodu ne sont jamais exactement constitués à I'aide des mêmes feuilles. De plus celles-ci sont certes choisies en fonction de leurs propriétés médicamenteuses, mais aussi surtout de leur valeur symbolique ou de l'impression qu'elles donnent compte tenu de leur forme, de la sensation que leur toucher procure, de l'aspect du tronc des arbres sur lesquels elles sont prélevées, etc... et du milieu (aquatique, de forêt, de savane...) où elles croissent.

Il y est ajouté d'autres ingrédients : têtes desséchées, morceaux de peau ou d'os d'animaux, cailloux singuliers, miniatures en fer forgé, en terre cuite ou en bois... dont la puissance dépend aussi d'un effet de forme ou d'un procédé particulier de fabrication.

- Dans de nombreux sanctuaires qui se sont récemment multipliés du culte de Mammi Watta, la puissance du vodu n'est pas constituée par des feuilles mais par une importante collection d'objets de luxe (parfums, poudres de beauté, savons, bijoux traditionnels et européens, bible...) qui, au lieu d'être enterrés, sont ostensiblement 
étalés sur une ou plusieurs tables. Une telle collection d'objets, disent les spécialistes du culte, tient lieu d'ama (plantes magicomédicinales) de Mammi Watta ${ }^{1}$.

- A en juger par les crises de possession qui se déclenchent lors des cérémonies de leur entretien, les "médicaments-vodu" déploient une activité d'autant plus intense que de nombreuses personnes y ayant placé leurs espoirs se trouvent rassemblées autour d'eux. En serait-il ainsi si leur puissance ne tenait qu'à celle des plantes dont ils se composent ? De toute évidence elle dépend très largement des convictions qui s'y attachent. A la manière de transformateurs spécialisés, ils ne font que restituer sous une autre forme celle qui leur est conférée.

En réalité la puissance en cause résulte plutôt d'une captation de l'attention par des objets ou des images exerçant matériellement ou symboliquement sur un être humain une sorte de fascination. Une fois délibérément entretenue, elle a ainsi même nature que celle des "objets" de concentration (diagrammes, statues, images mentales, idées...) à l'aide desquels des ascètes parviennent à développer, tant au réel qu'au figuré, de la "chaleur" intérieure. Elle tient au rassemblement d'une énergie vitale ou psychique autour de stimuli objectifs ou imaginaires faisant fonction de dzoka ("corde ou lien du feu"), énergie que le magicien s'emploie à "nouer", "attacher", ou mettre en paquet par une action assimilée à un "attachement du feu" (dzosasa). Elle est celle d'un investissement psychique ne pouvant pas davantage être saisi du côté d'un quelconque objet en soi que d'un quelconque sujet en soi, dont on ne comprendrait plus qu'ils interagissent, mais au niveau d'une relation fondamentale, antérieure aux termes qu'elle unit, constitutive de ceux-ci et dont ceux-ci ne sont jamais que l'illustration (les créatures d'un créateur), celle obligeant tout sujet à se mesurer avec le monde extérieur pour y réaliser ou y rencontrer, transformé en clair, l'objet irrémédiablement obscur de son véritable désir.

Fèves et jus végétaux avaient en effet autrefois même valeur que
Ies parfums. Quant aux ingrédients complémentaires, recueillis ou les parfums. Quant aux ingrédients complémentaires, recueillis ou
fabriqués, ils constituaient à des titres divers des objets rares et précieux analogues à d'actuels objets de luxe. 
Pour mieux en comprendre les propriétés il nous faut examiner comment les Evhé se représentent la participation des esprits à la grande oeuvre divine au niveau de laquelle ils entrent en compétition.

Nous trouvons répandue chez eux la notion d'un monde de la manifestation perceptible ou clairement intelligible, sorte de scène où se joue le drame de l'existence, localisé sous le ciel à la surface de la terre. Appelé kodzogbe, ce monde est l'aboutissement d'un processus de réalisation faisant passer une multitude de virtualités de la puissance à l'acte. Un phénomène réalisé est un phénomène fixé en sa forme définitive, ayant perdu en cours de route toute indétermination, arrivé au terme de sa traduction ou complète transposition par des esprits à partir d'indications demeurant sauvegardées au seuil du monde de l'origine (I'amedzophe).

Sous la direction générale du Créateur, ce sont les esprits (luvho) qui produisent le monde conformément aux engagements de leur existence prénatale. Cependant ils ont besoin pour cela de puiser dans 1'atmosphère où ils sont plongés une énergie fondamentale qui est celle d'un Souffle (gbogbo) cosmique. Chacun selon sa spécialité, ils se relayent indéfiniment pour entretenir dans un espace fini une somme de réalisations correspondant à la quantité d'énergie mise à leur disposition.

Là où elles aboutissent, leurs réalisations conservent avec elles, sous forme d'énergie concrétisée radicalement différente car cette fois mesurable, une partie de l'énergie cosmique fondamentale. Elles suivent dès lors automatiquement leur cours à la façon dont un mobile ayant été lancé adopte une trajectoire entièrement déterminée par les lois de la mécanique rationnelle.

L'énergie fondamentale, antérieure aux phénomènes, rend seulement les esprits producteurs de ces derniers entreprenants. Quant à l'énergie concrétisée, répandue au sein même des formes sensibles, elle se retrouve piégée dans le monde en tant qu'il continue d'être ce qu'il est d'un instant sur l'autre en dérivant ce qu'il devient de ce qu'il fut depuis un instant arbitraire de référence ou une origine absolue d'où il émergea comme par enchantement et dans le gouffre de laquelle il tend à nouveau à disparâttre. Elle préside à la continuité des événements d'un système apparemment clos au 
sein duquel rien ne se crée, rien ne se perd, tout se transforme par enchaînement selon des lois fixées à l'avance. Elle ne correspond qu'à la puissance de génération de ce qui est par ce qui fut, et non à une puissance de recréation de ce qui déjà est posé.

Il n'en reste pas moins que certains esprits ont la possibilité de modifier dans une certaine mesure les événements qu'ils entretiennent. En effet le fonctionnement du monde réel présente un certain jeu ; nul n'en peut prédire localement l'histoire. D'une part chance et malchance y viennent constamment compliquer l'existence des créatures. D'autre part une sorte de rayonnement destructeur, émané du principe de la sorcellerie (Na) et tendant à tout ramener au chaos comme à augmenter la multiplicité désordonnée, y est partout diffusé ; obligeant les structures à être perpétuellement adaptées et réparées, il dissuade les esprits de se désintéresser un seul instant du monde et se révèle en fait le grand complice de la vie. A jamais parfait et complet, le monde se transformerait en un corps mort abandonné dans le vide ; mais les esprits y gardent prise en s'insinuant dans toutes les zones d'imprédictibilité qui le traversent ${ }^{2}$. Sans la moindre dépense d'énergie concrétisée, et par conséquent sans qu'il y paraisse, il leur est possible (voir plus haut p. 269) d'y provoquer de façon concertée une multitude de micro-événements attribuables au hasard, engageant irréversiblement l'avenir. Leur population enveloppe le total des réalités perceptibles d'une sorte de nimbe depuis lequel ce qui lui échappe et se voit done rejeté dans un "au-delà" trouve néanmoins le moyen d'y intervenir. Elle constitue une sorte de champ organisationnel qui en surveille et en canalise par d'incessantes retouches le devenir.

Les esprits directement appliqués à produire le monde se comportent en fidèles exécutants des impulsions créatrices qu'ils reçoivent

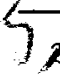

Indépendamment des variations atmosphériques, la trajectoire d'un obus est entièrement déterminée par la connaissance de son état à un instant antérieur. Au contraire, dans un jeu de pile ou face, un coup de rang supérieur à un n'est déterminé par aucun des précédents. En pratique ce sont les variations aléatoires infimes des conditions de son lancement et les fluctuations imprévisibles de I'atmosphère qui vont déterminer l'obus à tomber sur Monsieur X ou assez loin de lui pour ne pas le tuer. 
des profondeurs reculées de leur nature. Fournissant un effort ininterrompu d'étayage des phénomènes, ils manquent de recul pour songer à en modifier l'articulation et la succession. Seuls des esprits désincarnés, ou bien l'esprit imaginant (ku-luvho) qui double chez I'homme l'esprit vital (agbe-luvho) sont en mesure de s'y employer.

Les esprits désincarnés y parviennent à l'aide de la puissance spirituelle, ou énergie fondamentale d'incitation à l'action, conservée par certains d'entre eux ayant été victimes d'une mort brutale ou prématurée avant que leur vitalité n'ait été épuisée par l'âge ou la maladie. De tels esprits identifient diverses rémanences d'inclinations passées. Agissant isolément ou regroupés en bandes, ils ne provoquent habituellement que perturbation. Entrés toutefois au service des ancêtres, c'est-à-dire agissant en accord avec les causes finales adoptées par le milieu, ils contribuent, en assumant la basse besogne de ramener les récalcitrants dans le droit chemin, au renforcement de l'ordre social. Certains enfin font profiter directement de leurs connaissances et de leurs capacités des vivants ayant su pactiser avec eux.

Les esprits imaginants des vivants y parviennent à l'aide de la puissance spirituelle qu'ils doivent eux-mêmes fabriquer en évitant de dépenser leur énergie dans des productions objectives (de biens de consommation, de richesses, d'enfants...) ou des productions imaginaires (impliquant calcul, réflexions, supputations...au service des précédentes) pour la concentrer sur des rites, des paroles, des figurations, des idées...ne débouchant sur aucun nouvel acquis matériel ou mental, de nature à détourner le dynamisme vital sur des abstractions ou des symboles sauvegardés dans une étrange fixité de tout engagement dans le devenir, prenant ainsi figure d'objets de contemplation.

Accumulée en deça du monde de la manifestation, une telle puissance bénéficie, par rapport aux réalités définies qui se déterminent par enchaînement les unes avec les autres, d'une très large autonomie. Elle en demeure en effet retranchée et n'interfère éventuellement qu'avec des puissances de même nature ayant été pareillement élaborées ou se trouvant conservées après leur mort par d'autres esprits, 
disséminées dans l'espace de précipitation du sens sur les conduites humaines au travers duquel, comme au travers d'une atmosphère, il semble que celles-ci, depuis une origine transcendante (le lieu de fixation des mythes reporté par les Evhé au seuil de l'abîme d'où le soleil se lève), soient envoyées s'affirmer à même le monde des apparences. Correctement dominée par le sujet qui l'a produite, elle lui permet d'influencer directement d'autres esprits, incarnés ou désincarnés, en exerçant sur eux tel ou tel "charme". Incorrectement dominée par lui, elle attire à elle des esprits errants qui en prennent volontiers charge à sa place.

Un effort d'accumulation de puissance spirituelle est de ce point de vue recommandé à tout individu désireux d'élever sa conscience au plan des réalités subtiles ou divines. C'est en effet une intensification de son "souffle" non engagé qui le rend sensible à des esprits, incite des esprits (bons ou mauvais selon ses intentions) à s'intéresser à lui et donne du même coup aux divinités le moyen d'agir avec efficacité en lui.

\subsection{Notion de charme}

Découvertes autour d'eux ou élaborées à leur convenance par des hommes, les puissances spirituelles (gbogbo) agissent à l'état brut à la façon de médecines : elles créent des atmosphères favorables ou défavorables à la présence ou à tel ou tel type d'activité de certains esprits.

Il leur arrive d'être chevauchées, manipulées et consommées par des esprits désincarnés qu'elles attirent. Cependant les esprits imaginants des vivants ont aussi la possibilité de s'en emparer ou d'y garder fermement prise pour déformer localement l'espace au travers duquel se poursuit l'invisible gestation des événements et ainsi en bouleverser à leur profit l'arrangement définitif. Comme, ce faisant, ils ne dépendent du bon vouloir d'aucun autre esprit, ils obtiennent des résultats immédiats. On considère qu'ils exercent là un certain "charme". 
Moyen d'influencer à son insu un autre esprit, qu'il s'agisse de l'esprit d'une créature vivante ou d'un esprit désincarné, d'un esprit humain ou d'un esprit affecté à un autre règne de la nature, un charme (bo) se résume en une recette de captation ou de fabrication, d'entretien et d'utilisation dans un but précis d'une puissance spirituelle adéquate.

On séduit couramment par le vêtement, par la parure et par le langage, mais la même tenue, la même mimique et le même discours n'ont aucun effet sur le client, l'électeur ou la belle à conquérir s'ils ne sont pas employés avec conviction et si, adressés plusieurs fois à la même personne, ils ne varient pas quelque peu. Rapportée par un auditeur, la plaisanterie d'un grand comique perd énormément de valeur ; contrairement à la loi voulant que les mêmes causes produisent toujours, dans les mêmes circonstances, les mêmes effets, elle devient vite ennuyeuse à force de répétitions.

Un charme renvoie pareillement à un univers de significations. Il se formule dans un langage qui, au sens très large du terme, embrasse tous les moyens sensoriels de communication entre les êtres. Cependant il reste inopérant si son utilisateur n'y ajoute pas toujours un peu du sien et s'il ne prend pas pour moteur un "souffle" en sympathie avec lui et comme exalté par lui.

Gameli Toguna ("Critique de la représentation du destin chez les Evhé...", thèse de $3 e$ cycle, Paris III, Institut de Littérature Générale et Comparée, 1978), qui définit le bo comme "une force spirituelle opérante" (p. 107), une "force ésotérique" (p. 108), ou l'"expression d'une foi opérante" ( $p$. 115), nous l'assure inefficace à défaut des herbes sacrées employées et de "la disposition intérieure du guérisseur prononçant la parole (gbesa) appropriée au cas traité" (p. 109). En accord avec J. Spieth ("Die Ewe Stämme", Berlin, E. Vohsen, 1906, pp. 515-516) nous affirmant que "le dzo de chacun a son siège à l'intérieur de l'homme, surtout dans sa volonté et ses sentiments", il nous montre la "nécessité de la foi du détenteur de dzo, foi sans laquelle son bo n'a plus de valeur, devient une "chose simple" (p. 117).

De même que l'éducation sexuelle, celle du "langage" de l'amour charnel, est réalisable à l'école, mais non l'éveil de l'amour (à 
distinguer de l'attachement par investissement libidinal d'autrui), les techniques de fabrication et de mise en oeuvre d'un charme peuvent aisément être enseignées, mais non la foi, ou suranimation du coeur, ou participation à l'élan créatif universel, sans le soutien de laquelle il ne fonctionnerait pas. Celle-ci ne se communique que de personne à personne par un transvasement de maître à disciple, analogue au bouturage d'une plante. Tenter de l'obtenir par un effort de volonté ne conduit qu'à gesticuler isolément dans le vide. Elle ne se crée pas à partir de rien mais s'accroît après ensemencement ou rattachement à une filière. Ainsi est-elle apparentée à la vie dont on parvient à mâtriser la propagation et à déformer l'expression, sans que personne ait jamais encore découvert une recette permettant de la produire. L'irremplaçabilité de la relation humaine, et par conséquent de la transmission orale, apparaît en ce domaine évidente.

Un charme ne touche pas directement aux événements eux-mêmes. Il ne s'oppose en rien aux lois de notre monde, où ses effets restent donc impossibles à apprécier. Les paysans et artisans evhé qui y ont recours en concilient parfaitement l'usage avec la prudence et le sens des réalités qui les caractérisent. Ils ne lui attribuent d'effets que sur les agents spirituels (luvho) occupés à transformer en phénomènes sensibles les contenus obscurs d'une réalité fondamentale ou sur certains de ceux ayant seulement conservé après leur mort le pouvoir de modifier les processus de pareille transformation. Nous le voyons ainsi porter seulement à conséquence au niveau de l'induction des phénomènes par le champ organisationnel qui les enveloppe, induction possible en raison d'une intrusion de l'aléatoire dans tous les systèmes physiques et psychiques.

Grâce à eux I'homme échappe astucieusement à un enfermement dans le domaine de la causalité transitive (celui des causes matérielles et formelles faisant dériver le présent du passé). Cependant il échappe aussi à une sujétion complète à des entités supérieurs à lui, ancêtres et divinités, en mesure de modifier à la source même l'orientation des événements, qui représentent des puissances d'intégration en divers idéaux de ce qui subsiste du passé. En définitive il ne les collectionne qu'à proportion de sa croyance 
en la liberté de la personne humaine, dans l'intention de prendre activement part à la fixation de son histoire. Loin de correspondre à un stade d'embourbement de l'esprit dans ses peurs, ses illusions et ses contradictions, ils se situent dans une perspective d'accession de chacun à la pleine responsabilité de ses actes.

Une telle liberté se traduit négativement, il est vrai, par une inquiétante vulnérabilité aux "charmes" d'autrui, mais ne soyons pas rebutés outre mesure par cet inévitable revers de médaille. Nul ne se contente de la seule liberté de rêver ou de monologuer. Il aspire à une liberté réelle, c'est-à-dire confrontée à celle d'autrui, qui la rejoigne ou qui en triomphe. La liberté d'action ambitionnée (il n'est évidemment pas question ici de la liberté intérieure que procure le renoncement et le non-agir) implique une interdépendance générale des sujets ne les faisant exister qu'emportés dans un même mouvement d'attraction et d'opposition, d'unification et de distinction, dissuadant à jamais de les prendre en considération isolément.

Des magiciens compétents parviennent, à l'aide de charmes exerçant une contrainte sur des esprits errants désincarnés, à compléter leur propre puissance spirituelle par celle dont jouissent encore ces derniers. Comme de tels esprits sont acceptés en grand nombre au service des comités spécialisés d'ancêtres institués en divinités, ils parviennent même à y associer la puissance divine de certains de leurs ancêtres et des principes qu'ils représentent. Le "fétichisme" systématique des Evhé et autres populations de l'ancienne "Côte des Esclaves" a ainsi pour effet de rendre surpuissants les "objets" de culte par regroupement de tous les niveaux auxquels ils sont susceptibles d'appartenir. Les vodu qui les ont rendu célèbres et caractérisent au premier chef leur religion en sont le fruit le plus éminent. Loin de témoigner d'une déliquescence conceptuelle, le flou apparent du terme, servant à désigner ce qui est tout à la fois support matériel de charme, esprit dangereux et divinité, résulte en fait d'une respectable volonté de synthèse des moyens dont I'homme dispose pour échapper à la nécessité naturelle en touchant aux causes efficaces d'un monde dont il se sent prisonnier. 
A la différence des "médecines" n'exerçant qu'une influence locale diffuse, un charme est censé déclencher une projection orientée de puissance spirituelle. A cet effet il met en jeu des éléments intersubjectifs comme ceux du langage, des divers codes symboliques, de la représentation de l'espace, du schéma corporel, etc... qui occupent et structurent le référentiel culturel permettant une communication (transfert de significations et non simple interaction) entre les personnes. Soutenue par une puissance convenable, une forte volonté en prise avec de tels éléments semble produire des effets au niveau du référentiel lui-même ou, par ricochet, au niveau des gens dont les échanges avec le monde extérieur en dépendent. Elle affecterait spécialement ceux qu'un objet imprégné par eux met en rapport avec l'opérateur magicien ou par lesquels le corps du client de ce dernier se trouve lui-même imprégné. D'esprit à esprit est théoriquement opéré ainsi un transfert d'énergie fondamentale (non encore concrétisée) affectant les dispositions et le niveau d'activité de celui qui est visé.

Une telle énergie est de celles qui incitent les esprits à agir et les tissus des organes à respirer, c'est pourquoi je la qualifie le plus volontiers de spirituelle. La qualifier de psychique laisserait croire qu'elle n'est pas de même nature que celle qui vivifie les corps ; or elle ne devient psychique que du point de vue de certains de ses effets. La qualifier de vitale laisserait croire qu'en insufflant la vie elle disparất à la mort, alors qu'il n'en est rien. Elle appartient à une vie préexistante aux corps, propre à induire la formation des corps, et non à celle manifestée automatiquement par ceux-ci en raison d'un bel agencement de leurs parties. Elle n'est vraiment vitale qu'en matière de vie spirituelle dont un homme vulgaire, non né une seconde fois par l'initiation, notamment celle dispensée dans les "couvents vodu", reste privé.

Ses transferts, entre sujets mutuellement définis dans un même référentiel de communication, impliquent $1^{\prime}$ existence d'un champ de force et donc de forces que l'on pourrait qualifier de vitales en tant qu'elles poussent les êtres à la lutte pour la manifestation de leur nature, mais que je préfère qualifier aussi de spirituelles 
en tant qu'elles sont déployées par des esprits et donc attribuables à ces esprits plutôt qu'aux phénomènes, et notamment aux corps, entretenus par eux.

\subsection{Le champ de force spirituelle}

Les Evhé se représentent le milieu énergétique ou champ de force fondamental au sein duquel tous les esprits se trouvent plongés comme constitué par un Souffle créateur portant tout événement à venir se concrétiser à la surface de la terre. Le va-et-vient de la respiration n'est qu'un moyen pour chaque créature de capter une partie de ce dernier à son profit en l'attirant sur un circuit de dérivation.

Au débouché du monde de l'origine où s'élaborent, se décomposent en leurs éléments et se restructurent les causes finales des comportements, ce Souffle est accordé aux virtualités libérées de toute pression inhibitrice (celle exercée par les ancêtres et les gestionnaires divins de la création qui en ordonnent de concert l'actualisation) par la troisième composante du Dieu suprême (Mawu). Complétant son aspect mâle de Mâtre du Ciel et son aspect femelle de grand fixateur des destins appliqué aux fruits des entrailles de la Terre, cette composante, qui lui vaut le titre de Gbedhotó, en fait le dispensateur universel de la vie (agbe) sous ses formes perceptibles. Privées de son secours les deux premières ne serviraient à rien. Elle en est à la fois le produit (l'enfant du Père et de la Mère), le révélateur, ou continuateur, et la raison d'être ou plutôt l'aboutissement de la raison d'être. De même que le mélange de deux substances (par exemple une lumière blanche résultant du mélange de deux lumières complémentaires) évoque beaucoup mieux que chacune d'elles le principe d'unification les ayant forcées à se conjoindre, elle est malgré son rang inférieur la plus digne représentante de l'Un, ou principe suprême, qui assure I'unification des trois aspects de I'"Insurpassable" (Mawu).

A son tour le mâttre de la vie travaille à l'aide de deux puissances complémentaires, mâle et femelle, dont l'interaction engendre le Souffle. Ces deux puissances, d'où dérivent en conséquence tous 
les vodu, sont représentées par le soleil et par la lune 3 dont les mouvements différents ont pour effet d'imprégner l'atmosphère, comme le constatent les cultivateurs, d'incitations à l'action de diverses qualités. Selon une tradition largement répandue au sud de la boucle du Niger, antérieure à la prise en considération des sept planètes astrologiques, la périodicité luni-solaire réelle de 228 ans (ou 235 cycles de douze lunaisons), égale à 12 cyles de Méton de 19 ans, est jugée résulter d'une périodicité fondamentale inapparente de $7 \times 33=231$ "années lunaires" et les souffles spécifiques correspondants sont alors regroupés sous la présidence de sept grands principes vitaux représentés par des serpents mythiques de 33 vertèbres (de Surgy, La divination par les huit cordelettes chez les Mwaba-Gurma, Paris, L'Harmattan,1983, 102-112 et 116-122).

Ces principes, en lesquels le Principe Vital se décompose à la façon dont la lumière du jour se décompose en les "sept couleurs" de l'arc-en-ciel, sont connus sous le nom de Kla (Kra en langue akan). Ils président tour à tour aux sept jours de la semaine et méritent aux enfants d'être nommés d'après celui de leur naissance. Ce sont eux que de savantes compositions végétales aident en définitive à évoquer sous différentes sortes de figures dans le culte des vodu4.

Le champ de force of les esprits puisent l'énergie qui leur est nécessaire aussi bien à la production des phénomènes qui leur sont directement imputés qu'à une projection magique d'influence modifiant l'activité d'autres esprits nous apparaît donc septuplement qualifié, mais unifié par le radieux Créateur ayant su obliger les pires extrêmes (définissant initialement l'en-haut et l'en-bas) à contribuer à la production d'un seul et même univers, à savoir le principe du Souffle supportant toute manifestation et déployant dans la couverture

$63^{3}$ Théophanies du couple Lisa-Mawu qui, dans la mythologie des Fon, aurait engendré tous les vodu.

4 Les grands vodu se répartissent en trois catégories dont chacune

- se subdivise en trois familles : vodu célestes (So, Agbui, Da), vodu terrestres (Sakpata, Anana (Bluku), Gu), et Afa demeurant à proximité immédiate des hommes (Afa Dzisa, Nago, Tsaké). Mais chaque famille de vodu se compose théoriquement de sept vodu en rapport avec les sept principes vitaux. Bien que ceux-ci n'apparaissent qu'au dernier rang de l'analyse, les vodu ne sont jamais que des voies qui $y$ conduisent et leur sont tous redevables de leur efficacité. 
végétale du sol toutes les facettes de sa puissance, inévitablement inducteur de conceptions panthéistes.

En les absorbant en son sein, il rend les esprits étroitement solidaires, définissant pour eux un espace d'interaction immédiate ol ils tendent à s'unifier comme à l'intérieur d'un gel en tant que membres diversifiés d'un même Tout. Les charmes, éven'uellement signifiés par des objets ("fétiches") matériels utilisés pour les exercer, y jouent le rôle de supports des interactions et peuvent à ce titre être comparés aux quanta de champ, uniquement mis en évidence par leurs effets, des champs de force de la physique des particules élémentaires.

Complétant les dispositions de l'âme à vivre d'une certaine manière qui résultent de son existence prénatale dans l'amedzophe (celle-ci dépendant éventuellement de l'expérience accumulée au cours de précédents cycles de l'existence5), il lui donne les moyens de parvenir à ses propres fins, mais lui impose à cet effet une intégration dynamique au reste de l'univers que nous pouvons interpréter comme une participation à l'activité du "coeur" de celui-ci. Il en résulte pour elle une prolongation libératrice de perspective aboutissant, non plus seulement à une fatigante transposition en phénomènes d'un petit lot de virtualités, mais à l'Eveil de son propre coeur. Ce dernier se révèle en définitive essentiellement identique au principe suprême de vie et d'unification, ainsi que, par voie de conséquence, à tous ceux pareillement identiques en chacun des êtres.

Un tel champ induit non seulement les individus à se mesurer les uns aux autres, en situation de soumission ou de dominance, de complémentarité ou de rivalité, au niveau même des productions phénoménales dont ils se chargent, et pour ainsi dire par productions interposées, mais il leur permet d'entrer aussi en compétition au niveau du partage des énergies subtiles indispensables à de telles

Q5 J'appelle cycle d'existence la durée séparant l'envoi d'une âme dans le monde de dessus terre de son retour au ciel. Il comporte une existence prénatale souterraine, une existence manifeste sur terre et une existence invisible en tant qu'âme errante ou ancêtre. 
productions. Pour qui le prend en considération l'affrontement que nous jugeons "réel" entre les personnes du fait qu'il peut être objectivé par une mesure, mais qu'il vaudrait mieux qualifier de réalisé, avec la connotation de déjà passé ou de déjà mort que ce terme implique, n'est que le résultat du véritable affrontement qui se déroule sur un autre plan et que signale éventuellement un "fétiche". Les pourfendeurs de superstitions africaines auront beau jeu d'en nier I'existence ou d'en dénoncer la pernicieuse propriété d'inciter de pauvres gens à négliger les solutions effectives de leurs problèmes au profit d'une multitude de dérivations compensatoires dans I'imaginaire. Il ne peut en effet être décélé au niveau de la succession perceptible des événements ; mais, en bonne logique, cela ne devrait conduire qu'à le chercher au dessus d'eux, dans l'espace d'engendrement de la pression spirituelle qui s'y exerce. Qui donc, en fin de compte, se trouve "réellement" abusé ? Celui qui se détourne à l'occasion des phénomènes objectifs pour tenter d'agir directement sur les inclinations à l'activité d'autres esprits, ou celui qui imagine pouvoir négliger les conséquences de ces dernières ? Celui qui crô̂t en la liberté relative de l'homme, ou celui qui refuse d'en tenir compte ? La raison en la matière, n'est pas nécessairement du côté de qui croit en avoir l'exclusivité.

Du fait qu'il pousse les individus à entrer en rapport, peut-on qualifier ce champ de social ? Apparemment oui dans la mesure où, intermédiaire entre les choses et l'invisible nature où elles subsistent en puissance, il est d'emblée culturel ou est, du moins, culturellement structuré. Assurément non si on limite la portée du mot à ce que mesure la sociologie car il n'a de prise sur la réalité sociale qu'en ses zones d'incertitude ou à des moments d'instabilité dont aucune connaissance sociologique ou autre, celle de la façon dont les faits sociaux ou les autres faits intéressant la société sont engendrés les uns par les autres, ne permet de prévoir l'influence sur un système.

On peut admettre aisément que le domaine de la sociologie scientifique soit fondamentalement affecté d'une imprédictibilité analogue à celle reconnue de nos jours au sein de nombreux systèmes physiques. Nous entrons toutefois ici dans une dimension orthogonale à ce 
domaine, postulant sous le masque du hasard l'existence d'agents de décision extérieurs à ce qu'ils considèrent et manipulent, faisant fonction de véritables causes efficaces. Gouvernant aussi bien I'arrangement des phénomènes physiques que celui des phénomènes sociologiques, de telles causes ne sont pas plus sociales qu'autre chose ou, du moins, ne le sont que partiellement, du point de vue de certains de leurs effets. En nous y renvoyant, le "fétiche" apparaît, ainsi que le faisait remarquer Marie-Claude Dupré lors d'une réunion de notre équipe, jouer un rôle de point d'appui pour surmonter l'indétermination inhérente aux relations des individus avec leurs semblables et avec le monde. Cependant il ne conduit pas à envisager cette indétermination comme un élément de clôture sur lui-même (à l'aide de lois de probabilité) de l'univers phénoménal, mais comme une ouverture aménagée en son sein en direction de quelque transcendance, introduisant cette fois une véritable inexactitude dans la connaissance que nous en prenons.

\author{
Albert de Surgy \\ Laboratoire associé $n^{\circ} 221$ \\ EPHE - CNRS
}

Par rapport à la notation évhê officielle utilisée au Togo et au Ghana, nous avons adopté la transcription suivante : $0=0$, $v=v h$, $d=\mathrm{dh}, \varepsilon=\underline{e}, \quad \mathrm{~J}=\dot{\mathrm{n}}, \mathfrak{f}=\mathrm{ph}$ 


\section{THE INTERNATIONAL JOURNAL OF AFRICAN HISTORICAL STUDIES}

Forthcoming in Volume 20 (1987):

Women in Nationalist Struggle: Dar es Salaam's TANU Activists, by Susan Geiger

Slavery and Ideology: The South African Case, by R. L. Watson

Death in Zanzibar: Reflections on Slavery and Epidemiology, by Ibrahim Sundiata

Property and Theft in Kano at the Dawn of the Groundnut Boom, 1912-1914, by Allan Christelow

The Impact of Colonialism on a Nigerian Hill-Farming Society: A Case Study of Innovation among the Eggon, by David Dorward

Carrots, Sticks, and Cocoa Pods: The Spread of Cocoa Production in Assikaso, Ivory Coast, 1908-1920, by David Groff

Rural Political Protest: The Case of the 1953 Disturbances in Mweru-Luapula, by Mwelwa Musambachime

The Umarian Emigration of the Late Nineteenth Century, by David Robinson

Oromos, Slaves, and the Zar Spirits: A Contribution to the History of the Zar Cult, by Richard Natvig

Ordeal of an African Independent Church: The Nigerian Zionist Methodist Mission, 1942-1970, by Felix Ekechi

Subscription rates (per volume, published quarterly):

$\$ 53.00$ institutions and libraries, $\$ 25.00$ individuals. Back-issue rates available on request. To subscribe, make checks payable in U.S. funds to IJAHS - African Studies Center, and send to IJAHS Subscriptions, African Studies Center, Boston University, 270 Bay State Road, Boston, Massachuselts 02215 USA. 